\title{
Design of Sliding Mode Variable Structure Servo Driver Based on Hardware
}

\author{
Changfan Zhang, Na Liu, Han Wu and Jing $\mathrm{He}^{*}$ \\ College of Electrical and Information Engineering, Hunan University of \\ Technology, Zhuzhou Hunan, 412007, China \\ hejing@263.net
}

\begin{abstract}
Based on sliding mode control theory, in this paper a control method of tension control system in printing machine is presented. Firstly, a sliding mode controller for decreasing steady-state error is designed based on the servo motor model in the tension control system of printing machine, and the simulation verification is carried out in the MATLAB/Simulink. Further the hardware circuit is implemented through the design of operational amplifier to reduce the chattering of the system, and the trapezoidal wave is served as the input signal to reduce the overshoot of printing machine generated at the moment of start and stop, ensuring the smooth start and stop of the system. With the simulation results and the experimental results being consistent, it illustrates the correctness and practicality of the proposed method.
\end{abstract}

Keywords: Tension control; servo control; sliding mode controller; operational amplifier; simulation and experiment

\section{Introduction}

The shaftless drive printing machine has become the main equipment in the printing industry, and the polychromatic chromatography mode is widely used for printing in the modern packaging and printing. The shaftless chromatography is vulnerable to a variety of factors in the printing process, which leads to chromatography chromatism. The error caused by the characteristic change of substrate material (e.g., printing deformation of substrate material, drying deformation) and tension instability is the main cause of chromatography chromatism. The tension control is the difficult point and key point for the control of printing machine, and has significant influence on the quality of the printing product.

The traditional tension control system mainly adopts the traditional PID control. Because the traditional PID control method is affected by the torque change, linear change, mechanical loss of the transmission device and other factors, the absolute accuracy of the control is poor. Reference [1] proposed an intelligent fuzzy PID control method by absorbing the impact of external stimulus, which achieved the tension control with small steady-state error rate and small fluctuation ratio. In Reference [2], based on the theory of traditional PID controller and torque control, the double closed loops control algorithm of the speed control loop and the tension control loop was proposed. Although the PID control structure is simple and the theory is mature, its robustness is relatively poor.

Reference [3] designed a neural network fuzzy controller for the tension control of the rolling mill. Reference [4] proposed the fuzzy control algorithm for the tension control. Reference [5] adopted the neural network inverse control, to realize the decoupling control between the speed and the tension. Reference [6] introduced an artificial neural network method with back-propagation training algorithm to weaken the decoupling 
control between the speed and the tension. Reference [7] proposed a multivariate robust control method to suppress the disturbance and reduce the decoupling effect between the speed and the tension. Reference [8] proposed the robust control strategy to weaken the tension fluctuation. Despite that the robustness of these intelligent methods is better than the PID control, however, it is difficult to implement.

Compared with other control methods, the sliding mode control method has the advantages such as short stability time, fast speed, good dynamic performance, strong robustness and easy implementation. Reference [9-11] adopted different control methods in the sliding mode control to realize the tension control. Reference [12-16] are all based on the sliding mode control method with simulation verification conducted in the MATLAB/Simulink. Reference [17], [18] are based on the sliding mode control method, and DSP was used to carry out experimental verification. In these references, it performed simulation in MATLAB/Simulink, or DSP was used to conduct the experiment under the computer environment. However, the sliding mode control is a high-speed switching control, and the sampling period of the computer is limited, the calculated lag will produce the chattering for the system.

In this paper, we propose a tension control method based on sliding mode control and employ operational amplifier for the hardware circuit design. First of all, by adding continuous control unit in the conventional proportion switching controller, a sliding mode controller is designed to reduce steady-state error. To verify the design of the controller, simulation is carried out in the MATLAB/Simulink. Secondly, based on operational amplifier, the hardware circuit is designed. Differential circuit and analog multiplication circuit are used to design the controller circuit, and the trapezoidal wave is served as the input signal to reduce the overshoot of printing machine generated at the moment of start and stop, which guarantees the smooth start and stop of the system.

\section{System Description}

In the printing process, the tension of printing process is determined by the servo motor. The substrate material is fed into the printing unit by the feeding-in roller, then sent out by the pull roller after finishing the printing process, as shown in Figure 1.

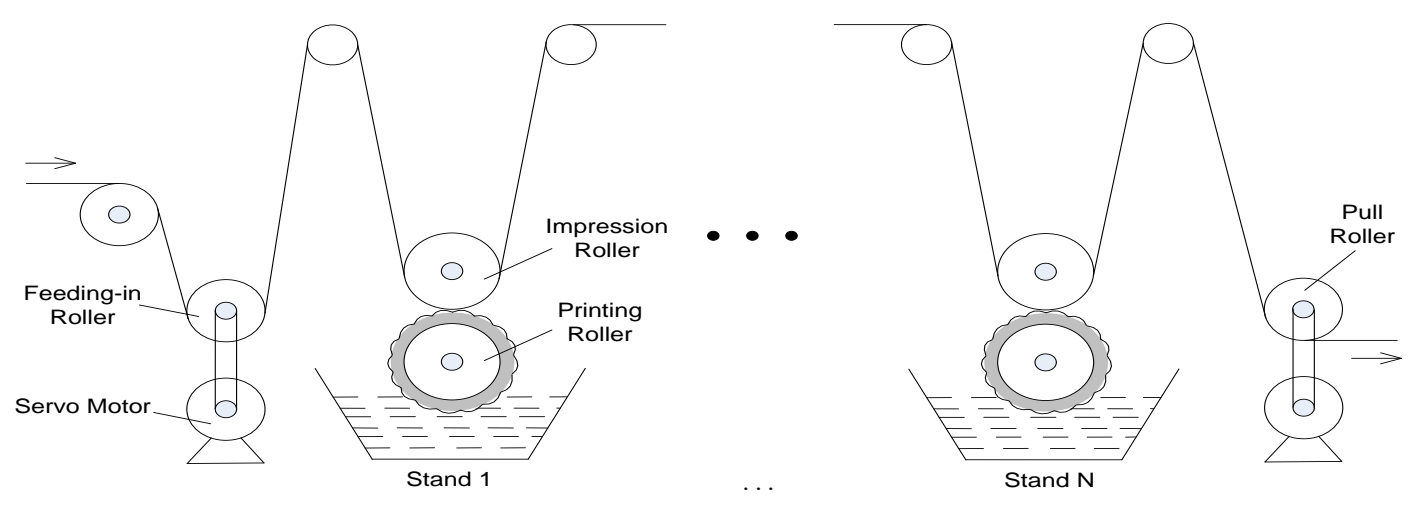

Figure 1. The Structure Diagram of Shaftless Drive Printing Machine

The feeding-in roller and pull roller accomplish paper feeding and paper delivery through the belt driven by the servo motor. The equivalent circuit of servo motor is shown in Figure 2. 


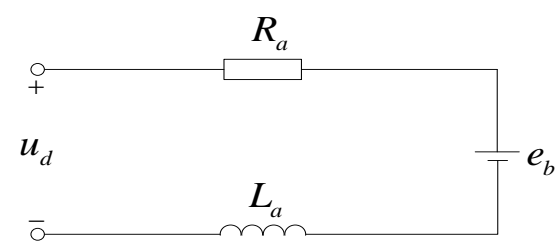

\section{Figure 2. The Equivalent Circuit Diagram of Servo Motor}

According to Figure 2, the transfer function of servo motor can be derived:

$$
\begin{aligned}
& G(s)=\frac{n}{u_{d}}=\frac{\frac{1}{C_{e}}}{T_{a} T_{m} s^{2}+T_{m} s+1} \\
& T_{a}=\frac{L_{a}}{R_{a}} \quad T_{m}=J \frac{R_{a}}{C_{m} C_{e}}
\end{aligned}
$$

with notations shown as follows :

$u_{d}$ : Armature voltage ;

$n$ : Motor velocity ;

$R_{a}:$ Armature resistance ;

$L_{a}:$ Armature inductance ;

$e_{b}:$ Counter electromotive force ;

$C_{m}$ : Electromagnetic torque coefficient ;

$C_{e}:$ Voltage feedback coefficient ;

$T_{a}$ : Electromagnetic time constant ;

$T_{m}$ : Electromechanic time constant ;

$J$ : Rotor moment of inertia.

Let $a_{1}=C_{e} T_{a} T_{m}, \quad b=C_{e} T_{m}, \quad a_{2}=C_{\mathrm{e}}$, Equation (1) is simplified as

$$
G(s)=\frac{1}{a_{1} s^{2}+b s+a_{2}}
$$

The above transfer function of the motor is transformed into the state equation as follows:

$$
\left\{\begin{array}{l}
\dot{x}_{1}=x_{2} \\
x_{2}=\ddot{r}-\ddot{y}=\ddot{r}+\frac{1}{a_{1}}\left[b \dot{y}+a_{2} y-u(t)\right]
\end{array}\right.
$$

where the state variable $x_{1}$ is the error between the given value and actual output value, i.e., $x_{1}=e=r-y$, and $x_{1}$ is represented by $V_{e}$ in the hardware circuit of this paper. 


\section{Design of Sliding Mode Controller}

The sliding mode surface of the controller is selected as

$$
s=c_{1} x_{1}+x_{2} \quad\left(c_{1}>0\right)
$$

where $c_{1}$ is the slope of sliding mode surface, to be designed.

The proportion switching method is adopted to design the sliding mode controller as

$$
u=\psi_{1} x_{1}+M \quad \psi_{1}= \begin{cases}\alpha_{1} & x_{1} s>0 \\ \beta_{1} & x_{1} s<0\end{cases}
$$

$$
M=a_{1} \ddot{r}+b \dot{r}+a_{2} r
$$

where $\psi_{1} \cdot x_{1}$ is the switch control unit; $M$ is the continuous control unit.

According to the formula (3), (4) and (5), we have:

$$
\dot{s}=\left(c_{1}-\frac{b}{a_{1}}\right) s+\left(-c_{1}^{2}+\frac{b}{a_{1}}-\frac{a_{2}}{a_{1}}\right) x_{1}-\frac{1}{a_{1}} u(t)+\ddot{r}+\frac{b}{a_{1}} \dot{r}+\frac{a_{2}}{a_{1}} r
$$

Lyapunov function is selected as

$$
V=\frac{1}{2} s^{2}
$$

$\dot{V}=s \dot{s}$

$$
=\left(c_{1}-\frac{b}{a_{1}}\right) s^{2}+\left[\left(-c_{1}^{2}+\frac{b}{a_{1}}-\frac{a_{2}}{a_{1}}\right) x_{1}-\frac{1}{a_{1}} u(t)+\ddot{r}+\frac{b}{a_{1}} \dot{r}+\frac{a_{2}}{a_{1}} r\right] s
$$

In order to ensure that the whole system is under the establishment condition of sliding mode, Equation (8) has to satisfy that $\dot{V} \leq 0$, namely the constraints in the following must be satisfied:

$$
\begin{gathered}
c_{1}<\frac{b}{a_{1}} \\
\alpha_{1}>a_{1}\left(-c_{1}^{2}+\frac{b}{a_{1}}-\frac{a_{2}}{a_{1}}\right) \\
\beta_{1}<a_{1}\left(-c_{1}^{2}+\frac{b}{a_{1}}-\frac{a_{2}}{a_{1}}\right)
\end{gathered}
$$

When the system parameters change, as long as the constraints described in Equation (9) are still satisfied, the establishment condition of sliding mode can still be satisfied. Therefore, this design method has relatively strong robustness.

\section{Implementation of Hardware Circuit}

In order to avoid the system discretization, the hardware circuit designed in this paper is realized by integrated operational amplifier. According to the design of sliding mode controller in Section 3, the implementation block diagram of hardware circuit is shown in Figure 3. All the operational amplifiers used in the control system circuit are chips LM358, and the power supply of the chip is dual power supply of $\pm 12 \mathrm{~V}$. 


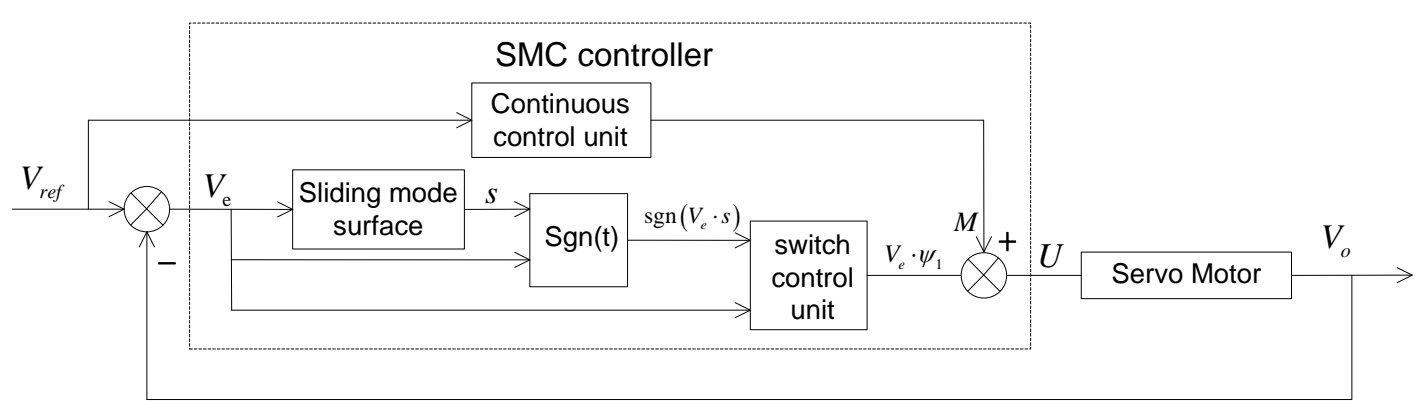

Figure 3. The Block Diagram of Sliding Mode Control

The hardware circuit of the controller consists of four parts: sliding mode surface, sign function, switch control unit and continuous control unit. We will describe them in the following subsections.

\subsection{Implementation of Sliding Mode Surface}

The hardware circuit of sliding mode surface is shown in Figure 4, where (1) is differential circuit, $R_{2} \cdot C_{1}=\frac{1}{f}$ ( $f$ is the frequency of input signal), and $R_{1}=\frac{R_{2}}{10}$. Therefore we have $s=-\left(\frac{R_{5}}{R_{3}} \cdot\left(-V_{e}\right)+\frac{R_{5}}{R_{4}} \cdot\left(-\dot{V}_{e}\right)\right)=\frac{R_{5}}{R_{3}} \cdot V_{e}+\frac{R_{5}}{R_{4}} \cdot \dot{V}_{e}$. According to Equation (4), we have $R_{5}=R_{4}$ and $\frac{R_{5}}{R_{3}}=c_{1}$.

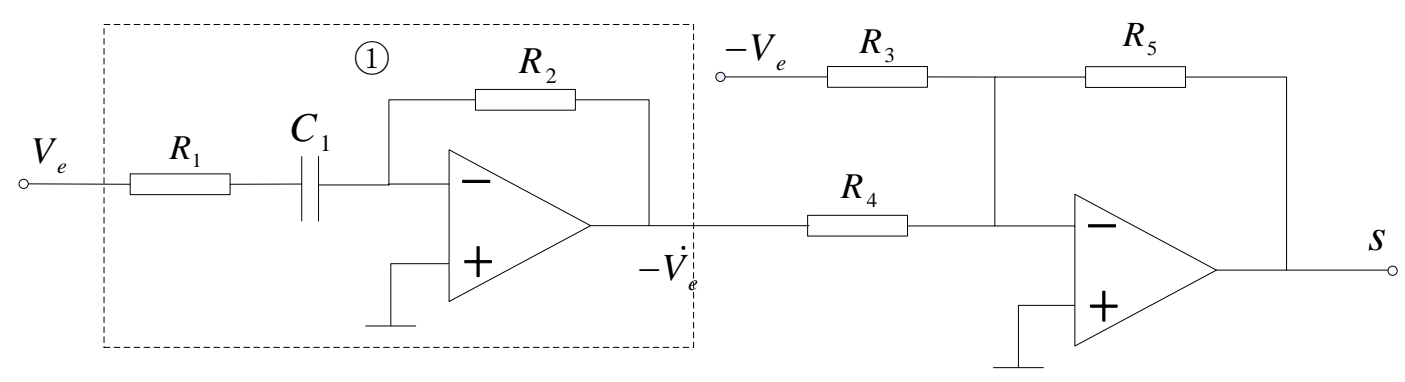

Figure 4. The Hardware Circuit of Sliding Mode Surface

\subsection{Implementation of Sign Function}

The hardware circuit of sign function is shown in Figure 5, where (1) is analog multiplier module constructed by chip AD835, the power supply of the chip is dual power supply of $\pm 5 \mathrm{~V}$ and (2) is zero-crossing comparator. When the voltage $V_{e} \cdot s$ is larger than 0 , the high level output is positive voltage of power supply; when the voltage $V_{e} \cdot s$ is less than 0 , the low level output is negative voltage of power supply. But the output of sign function is $\pm 1 \mathrm{~V}$, and the supply voltage of operational amplifier is $\pm 12 \mathrm{~V}$, therefore an operational amplifier circuit with shrinkage of 12 times is connected behind the zerocrossing comparator, as shown in (3) in Figure 5. 


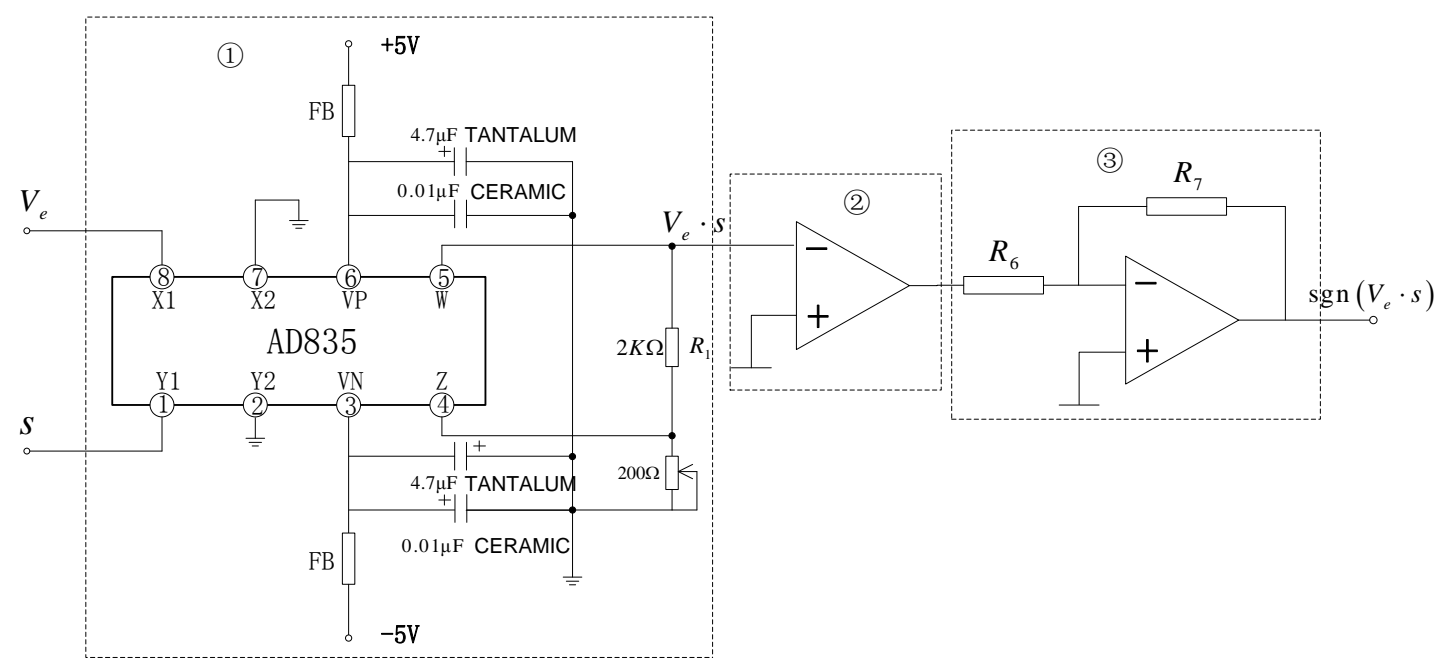

Figure 5. The Hardware Circuit of Sign Function

\subsection{Implementation of Switch Control Unit}

The hardware circuit of switch control unit is shown in Figure 6, where (1) and (2) are the implementation of $\psi_{1}$. In part (1) we have $\frac{R_{9}}{R_{8}}=-\psi_{1}$, and part (2) is the inverter circuit. When $\operatorname{sgn}\left(V_{e} \cdot \psi_{1}\right)$ is $+1 \mathrm{~V}$, the output of (1) and (2) is $\psi_{1}=\alpha_{1}$; when $\operatorname{sgn}\left(V_{e} \cdot \psi_{1}\right)$ is $-1 \mathrm{~V}$, the output of (1) and (2) is $\psi_{1}=\beta_{1}$. The hardware circuit of analog multiplier in Figure 6 is shown in (1) of Figure 5.

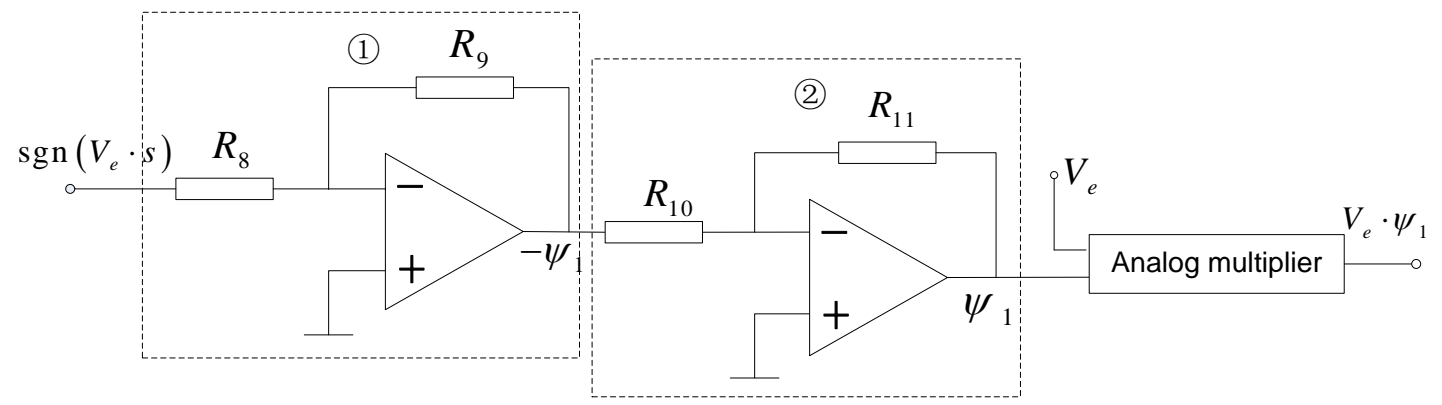

Figure 6. The Hardware Circuit of Switch Control Unit

\subsection{Implementation of Continuous Control Unit}

The hardware circuit of continuous control unit is shown in Figure 7. According to Equation (5), in this paper the given signals of control system are rectangular wave, skew wave, triangular wave and trapezoidal wave, and the second order derivative of these signals is 0 , therefore we have $M=b \dot{r}+a_{2} r$. (1) in Figure 7 is differential circuit, which is the same as that of hardware circuit of sliding mode surface in Section 4.1. (2) in Figure 7 is the implementation of $M=b \dot{r}+a_{2} r$, where $\frac{R_{16}}{R_{14}}=a_{2}$ and $\frac{R_{16}}{R_{15}}=b$. 


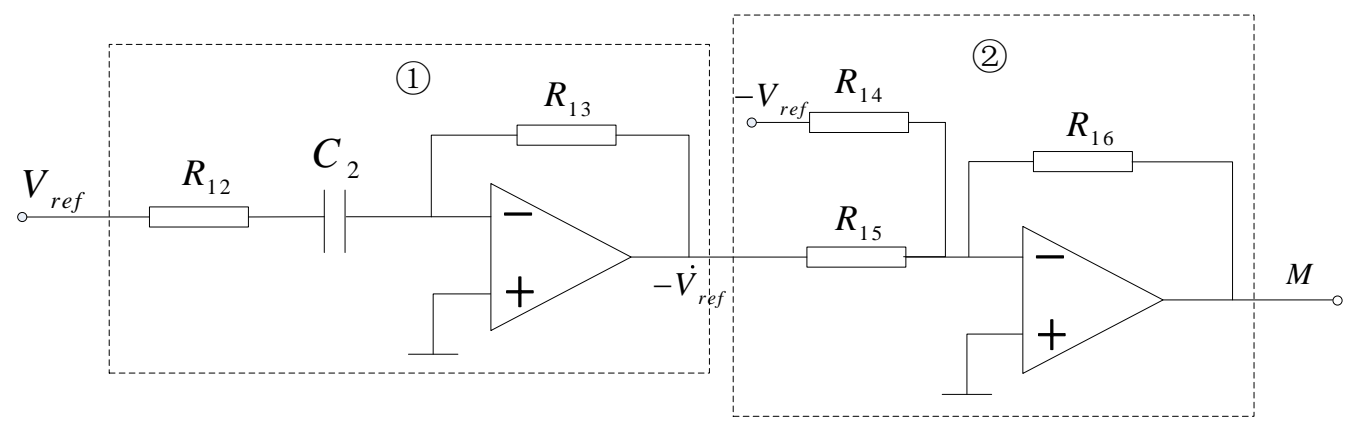

Figure 7. The Hardware Circuit of Continuous Control Unit

\section{Simulation and Experimental Results}

\subsection{Simulation Results}

To evaluate the proposed method, we conduct simulation experiment in the MATLAB/Simulink Software, and the parameters of servo motor are shown in Table 1:

Table 1. Nominal Value for Servo Motor

\begin{tabular}{c|c|c}
\hline \hline Parameters & Unit & Values \\
\hline Armature resistance $\left(R_{s}\right)$ & $\Omega$ & 7.77 \\
\hline Armature inductance $\left(L_{a}\right)$ & $H$ & 0.0777 \\
\hline Rotor moment of inertia $(J)$ & $\mathrm{kg} \cdot \mathrm{m}^{2}$ & 0.6 \\
\hline $\begin{array}{c}\text { Electromagnetic torque } \\
\text { coefficient }\left(C_{m}\right)\end{array}$ & $\mathrm{Nm} / \mathrm{A}$ & 6 \\
\hline $\begin{array}{c}\text { Voltage feedback coefficient } \\
\left(C_{e}\right)\end{array}$ & $\mathrm{V} /(\mathrm{r} / \mathrm{min})$ & 1.2 \\
\hline \multicolumn{2}{|l}{}
\end{tabular}

According to the Equation (1), we have $T_{a}=10^{-2}$ and $T_{m}=0.6475$; according to the Equation (2), we have $a_{1}=0.00777, b=0.777, a_{2}=1.2$, and further $\frac{b}{a_{1}}=100$ and $\frac{a_{2}}{a_{1}} \approx 154$. In the controller designed in this paper, the selected slope of sliding mode surface is $c_{1}=20$, and the parameters of the controller are $\alpha_{1}=10, \beta_{1}=-10$.

In the simulation, four signals of rectangular wave, skew wave, triangular wave and trapezoidal wave are served as the input signal. The simulation results are shown in Figure 8-11, respectively. 


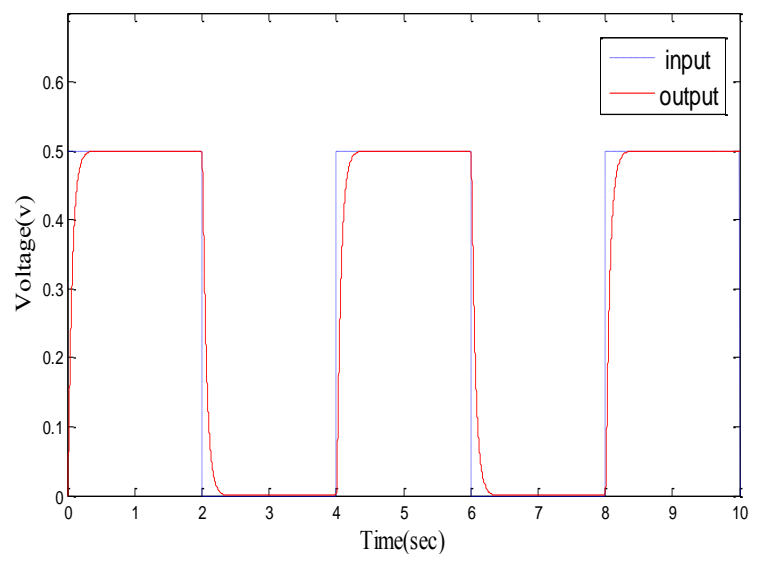

Figure 8. The Simulated Result of Rectangular Wave with Input Being 0.5V

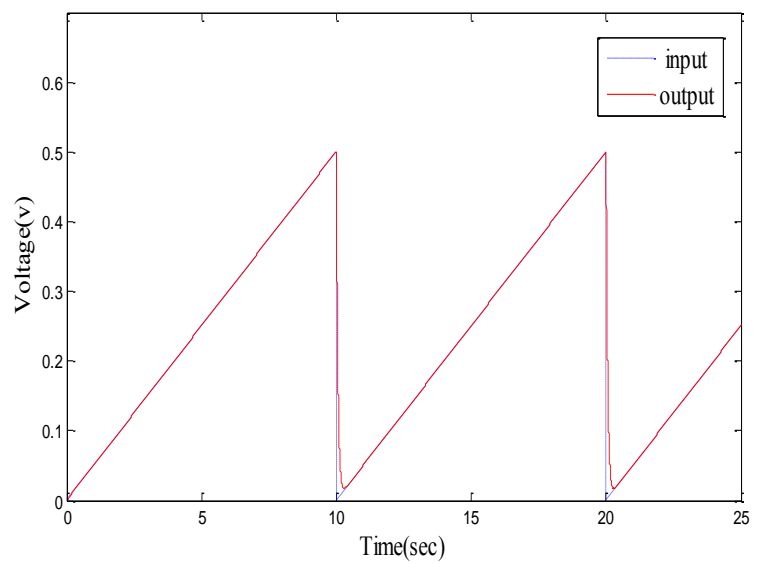

Figure 9. The Simulated Result of Skew Wave with Input Being 0.5V

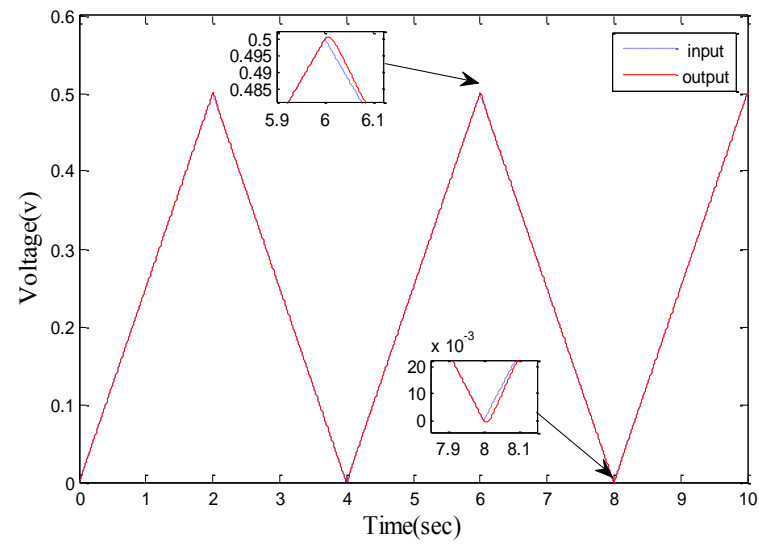

Figure 10. The Simulated Result of Triangular Wave with Input Being 0.5V 


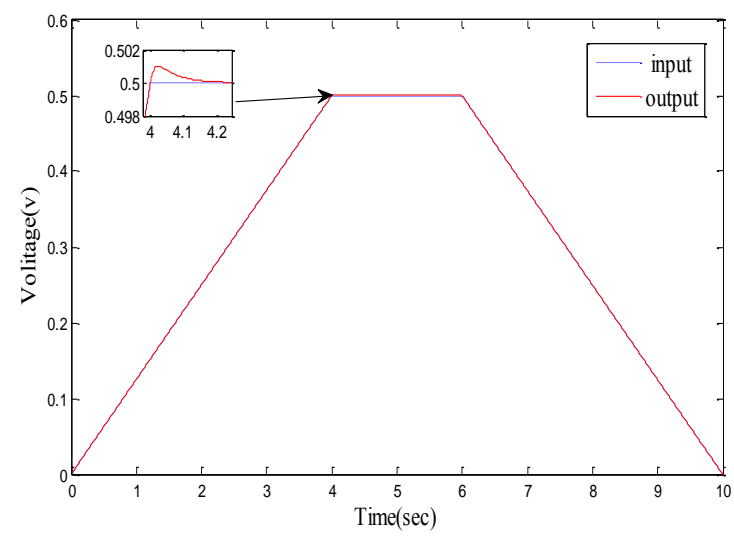

\section{Figure 11. The Simulated Result of Trapezoidal Wave with Input Being 0.5V}

Figure 8 is the simulation result when the input signal is the rectangular wave of $+0.5 \mathrm{~V}$, from the waveform chart we can see that at the instant of rising time and falling time of rectangular wave, the output voltage of servo motor does not follow the input voltage completely, but then it follows quickly. Figure 9 is the simulation result when the input signal is the skew wave of $+0.5 \mathrm{~V}$, in the waveform chart at the falling time of skew wave, the output voltage of servo motor does not follow the input voltage completely in the simulation. Figure 10 is the simulation result when the input signal is the triangular wave of $+0.5 \mathrm{~V}$, where in the process from rising stage to falling stage of the triangular wave, the servo motor appears slight overshoot in the simulation. Figure 11 is the simulation result when the input signal is the trapezoidal wave of $+0.5 \mathrm{~V}$, where in the process from acceleration stage to constant speed of trapezoidal wave, the servo motor appears slight overshoot in the simulation.

\subsection{Experimental Results}

To evaluate the hardware circuit design, we conduct experiments on the designed experiment platform, as shown in Figure 12.

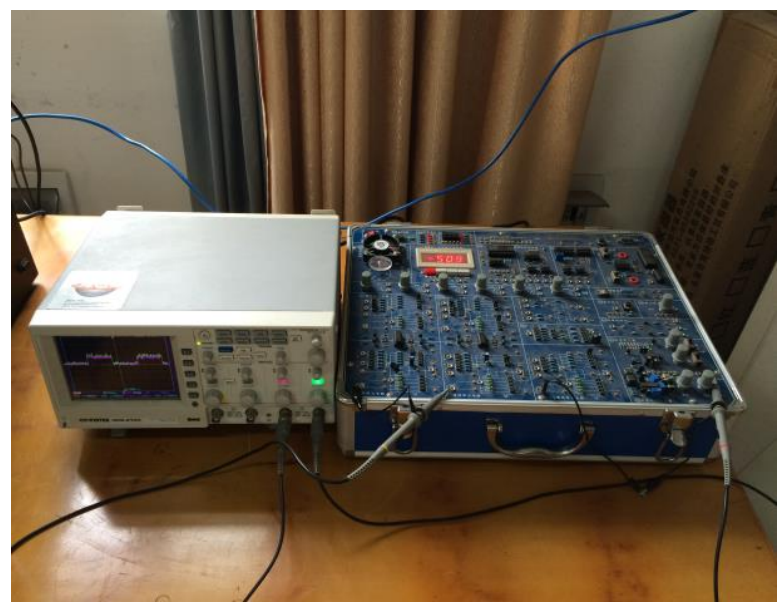

Figure 12. The Experiment Platform

According to the controller designed in Section 3 and the hardware circuit designed in Section 4, we configure the experiment as following:

$R_{1}=R_{12}=6.2 \mathrm{~K} \Omega, R_{2}=R_{13}=62 \mathrm{~K} \Omega, C_{1}=C_{2}=10 \mu \mathrm{F}, R_{3}=10 \mathrm{~K} \Omega$, 


$$
\begin{aligned}
& R_{4}=R_{5}=200 K \Omega, R_{6}=120 \mathrm{~K} \Omega, R_{7}=10 \mathrm{~K} \Omega, R_{8}=25 \mathrm{~K} \Omega R_{9}=250 \mathrm{~K} \Omega \\
& R_{10}=R_{11}=100 \mathrm{~K} \Omega, R_{14}=100 \mathrm{~K} \Omega R_{15}=150 \mathrm{~K} \Omega R_{16}=120 \mathrm{~K} \Omega .
\end{aligned}
$$

In the experiment, four signals of rectangular wave, skew wave, triangular wave and trapezoidal wave are respectively served as the input signal. The experimental results are shown in Figure 13-16.

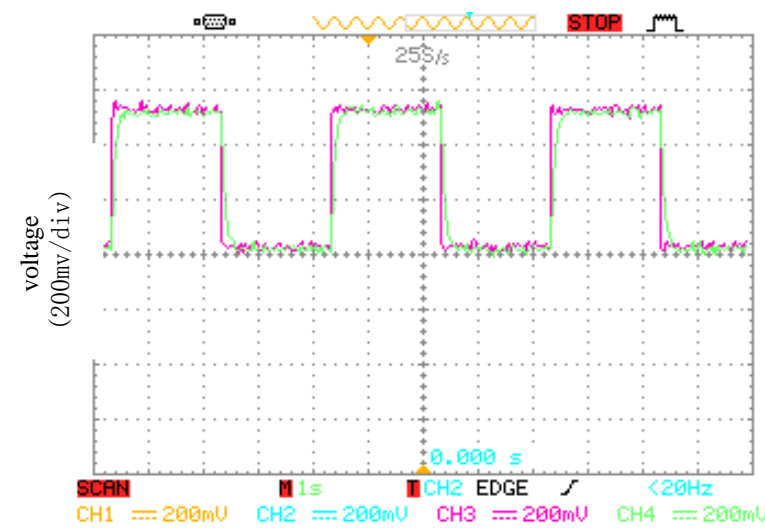

Figure 13. The Experimental Result of Rectangular Wave with Input Being 0.5V

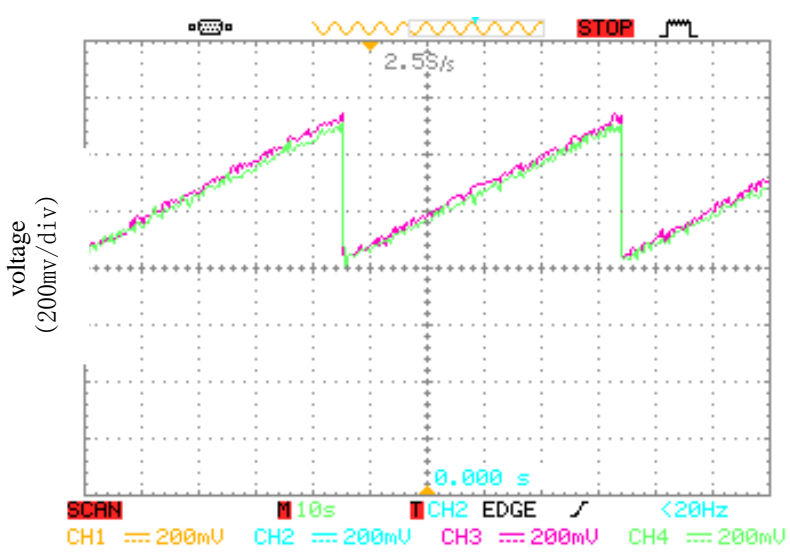

Figure 14. The Experimental Result of Skew Wave with Input Being 0.5V

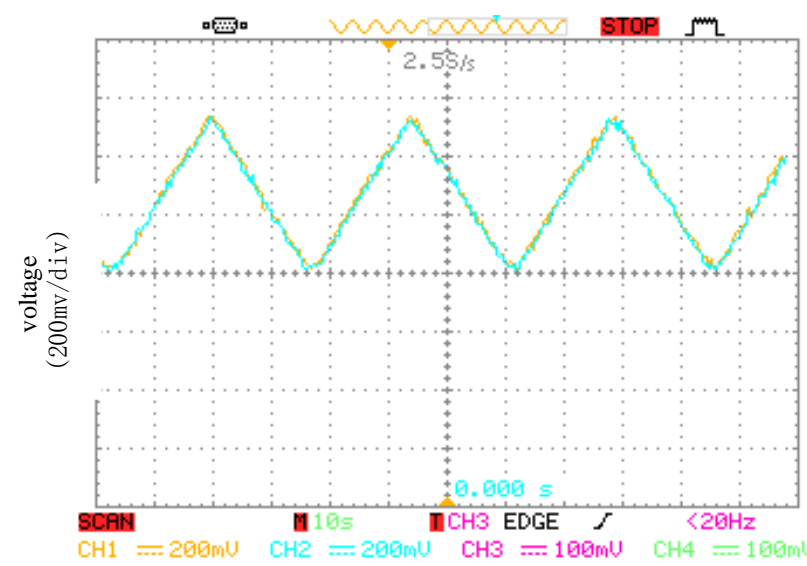

Figure 15. The Experimental Result of Triangular Wave with Input Being 0.5V 


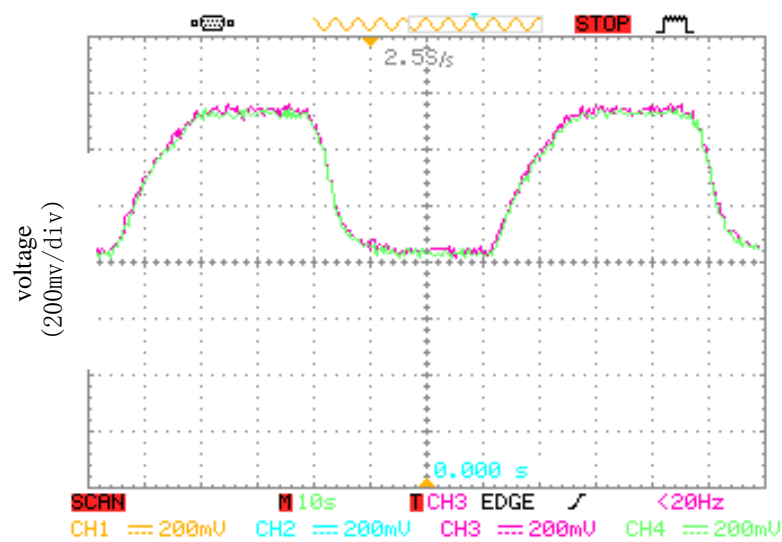

Figure 16. The Experimental Result of Trapezoidal Wave with Input Being 0.5V

As shown in Figure 13, where the input signal is the rectangular wave of $+0.5 \mathrm{~V}$, the output voltage of servo motor does not follow the input voltage completely at the instant of rising time and falling time of rectangular wave, but it follows quickly. The experiment results with input signal being skew wave of $+0.5 \mathrm{~V}$, triangular wave of $+0.5 \mathrm{~V}$ and trapezoidal wave of $+0.5 \mathrm{~V}$ are shown in Figure 14, Figure 15 and Figure 16, respectively. In each of the three cases, the output voltage of the servo motor follows the input voltage completely in the experiment.

Through comparing the simulation results in Figure 8-11 with the experimental results in Figure 13-16, it shows that the simulation results are identical with the experimental results, which verifies the rationality and practicability of this controller design. Meanwhile, through comparing the simulation results in Figure 9-11 with the experimental results in Figure 14-16, it proves that this hardware circuit reduces the chattering of the system.

\section{Conclusion}

In this paper, aiming at the control of servo motor in the shaftless drive printing machine, the controller based on the sliding mode control servo motor is designed. While simulation verification carried out in the MATLAB/Simulink, its hardware circuit is designed based on the mathematical model of the controller to reduce the chattering of the system, and this circuit is implemented for experimental test. With the simulation results being identical with the experimental results, it shows the correctness and practicability of this control circuit, and proves that this hardware circuit reduces the chattering of the system. Meanwhile, according to the simulation and experiment, with this controller the servo motor can achieve good performance that the output voltage follows the input voltage when the trapezoidal wave is served as the input signal, which guarantees the smooth start and stop of the system. In the sliding mode control, we only consider the proportion switching control in this paper and leave other methods into future work.

\section{Acknowledgements}

This work was supported by the Natural Science Foundation of China (nos. 61273157 and 61473117), Hunan Provincial Natural Science Foundation of China (nos. 14JJ5024 and 2015JJ5011), and Hunan Province Education Department (nos. 12A040 and 13CY018).

*Corresponding author: Jing He. 


\section{References}

[1] G. Li, "Research and application of intelligent fuzzy PID controlling press tension", China Printing and Packaging Study, vol. 05, (2009), pp. 48-52.

[2] M. Mu and S. Cao, "Research on tension control system of web press based on motion control", China Printing and Packaging Study, vol. 03, (2014), pp. 44-51.

[3] F. Janabi-Sharifi, "A neuro-fuzzy system for looper tension control in rolling mills", Control engineering practice, vol. 13, no. 1, (2005), pp. 1-13.

[4] F. Ru, Y. Ru and F. Hu, "Research on tension control technology based on fuzzy control algorithm", Electric Machines and Control), vol. 0, (20112, pp. 89-93.

[5] D. Wang, P. Ju and G. Liu, "Decoupling control of two motors system based on neural network inverse system”, Chinese Journal of Mechanical Engineering, vol. 17, no. 4, (2004), pp. 602-605.

[6] S. H. Jeon, J. M. Kim, K. C. Jung, S.K. Sul and J. Y. Choi, "Decoupling control of bridle rolls for steel mill drive system", In Industry Applications Conference, 1997.Thirty-Second IAS Annual Meeting, IAS'97., Conference Record of the 1997 IEEE, IEEE, vol.3, (1997), pp.2144-2150.

[7] D. Knittel, E. Laroche, D. Gigan and H. Koc, "Tension control for winding systems with two-degreesof-freedom $\mathrm{H}_{\infty}$ controllers. Industry Applications”, IEEE Transaction on, vol. 39, no. 1, (2003), pp. 113120.

[8] X. Dou and W. Wang, "Robust control of multistage printing systems", Control Engineering Practice, vol. 183, (2009).

[9] M. Yang, "Application and simulation of sliding mode control in winding tension control system", Packaging Engineering, vol. 03, (2013), pp. 44-48.

[10] H. Wei, G. Zhou and D. Shen, "Multi-motor Synchronous Control Strategy of Electronic Shaft Driving in Gravure Printing Machine", Packaging Engineering, vol. 03, pp. 134-139, (2015).

[11] S. Gong and N. Yang, "Chain tension control of scraper conveyor based on sliding mode control", Industry and Mine Automation, vol. 02, (2015), pp. 57-60.

[12] C. Zhang, Y. Wang, J. He and Y. Long, "GA-NN-integrated sliding-mode control system and its application in the printing press", Control Theory \&Applications, vol. 02, (2003), pp. 217-222.

[13] Y. Ma, "Research on synthetic control strategy of shaftless chromatography system", Northeastern University, (2010).

[14] J. Zhu, M. Su, X. Wang and L. Ma, "Direct-torque-control of brushless DC motors based on segmented sliding-mode-variable-structure”, Chinese Journal of Scientific Instrument, vol. 11, (2013), pp. 26342640.

[15] J. Yang, Y.-T. Liu, J. Xie, H. Liu and J. Wu, "Direct-torque control of the brushless double-fed machines based on sliding-mode variable structure control strategy", Control Theory \& Applications, vol. 09, (2011), pp. 1195-1201.

[16] J. Le, Y. Xie, Q. Hong, Z. Zhang and L. Chen, "Sliding mode control of boost converter based on exact feedback linearization", Proceedings of the CSEE, vol. 30, (2011), pp. 16-23.

[17] Z. Li, G. Hu, J. Cui and G. Liu, "Sliding-mode variable structure control with integral action for permanent magnet synchronous motor", Proceedings of the CSEE, vol. 03, (2014), pp. 431-437.

[18] Mates and Jose, "Feedback linearization of a single-phaseactive power filter via sliding mode control", Power Electronics, IEEE Transactions on, vol. 23, no. 1, (2008), pp. 116-125.
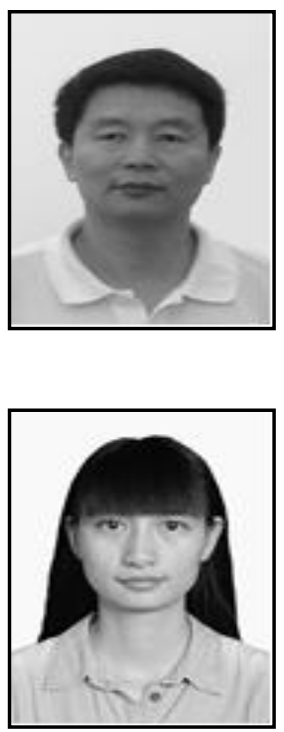

\section{Authors}

Changfan Zhang, he received the M.S. degree from Southwest Jiaotong University in 1989and the Ph.D. degree from Hunan University, China, in 2001. He is currently a Professor at College of Electrical and Information Engineering; Hunan University of Technology.His research interests include fault diagnosis on electric machines and industrial process control.

Na Liu, she graduated from Hunan University of Technology, China, in 2013. She is currently a graduate student at the College of Electrical and Information Engineering, Hunan University of Technology. 


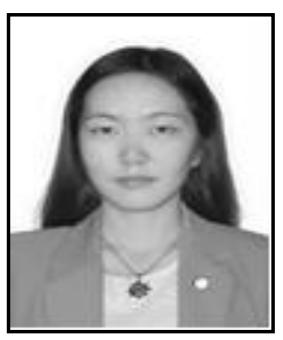

Han Wu, she graduated from Hunan University of Technology, China, in 2015. She is currently a doctoral student at the Department of Automation, Huazhong University of Science and Technology.

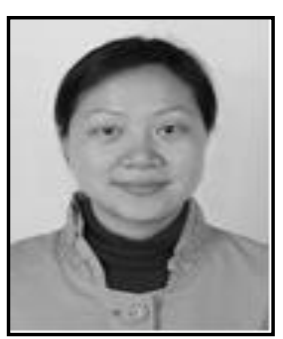

Jing He, she received the M.S. degree from Central South University of Forestry and Technology in 2002 and the Ph.D. degree from National University of Defense Technology, China, in 2009. She is currently a Professor at the College of Electrical and Information Engineering, Hunan University of Technology. Her research interests include fault diagnosison mechatronics and industrial process control. 
International Journal of Control and Automation

Vol. 10, No. 2 (2017) 